\title{
Distribución de EPA-PAH en los productos de la pirolisis de purín de cerdo
}

\author{
Pilar de Blas ${ }^{1}$, Iñaki Adánez-Rubio ${ }^{1}$, María U. Alzueta ${ }^{1}$, Gloria Gea ${ }^{1}$, Isabel Fonts ${ }^{1,2}$ \\ ${ }^{1}$ Grupo de procesos termoquómicos (GPT) \\ Instituto de Investigación en Ingeniería de Aragón (I3A) \\ Universidad de Zaragoza, Mariano Esquillor s/n, 50018, Zaragoza, Spain. \\ Tel. +34-976762707, e-mail: iadanez@unizar.es \\ ${ }^{2}$ Área de Química y Medio Ambiente \\ Centro Universitario de la Defensa (CUD) de Zaragoza \\ Academia General Militar, Carretera de Huesca s/n, 50.090, Zaragoza, Spain
}

\section{Resumen}

En este trabajo se plantea la obtención de biochar mediante pirólisis de purín de cerdo/porcino, como material de enmienda de suelos, en dicha aplicación, una limitación del uso del biochar es su contenido en hidrocarburos policíclicos aromáticos (PAH), debido a su toxicidad. Por ello, en este proyecto se estudia la formación de PAH durante la pirólisis de de purín de cerdo, evaluando su distribución en los productos de reacción: gas, líquido y biochar.

\section{Introducción}

El purín es un residuo orgánico generado en las explotaciones ganaderas, mezcla de las deyecciones sólidas y líquidas junto con restos de alimentos, agua de los abrevaderos, aguas de lavado y, en ocasiones, agua de lluvia. España es el segundo mayor productor de porcino en Europa, generando por tanto una gran cantidad de residuos. Es posible reutilizar este residuo empleándo el biochar generado en su pirólisis como enmienda en los suelos. Duante un proceso de pirólisis, es decir calentamiento en ausencia de aire, se dan unas condiciones favorables para la generación de compuestos aromáticos policíclicos, PAH [1]. Es, por tanto, importante estudiar cuál es la concentración que se obtiene y la distribución de los mismos en los productos.

Los compuestos aromáticos policíclicos (PAH's) incluyen un gran conjunto de compuestos que se generan durante la pirólisis o combustión incompleta de materia orgánica que contiene carbono e hidrógeno. Estos compuestos son potencialmente tóxicos y carcinogénicos. La Agencia de Protección Ambiental de Estados Unidos (EPA) ha clasificado 16 PAH (EPA-PAH) como contaminantes prioritarios debido a su potencial carcinogénico y su abundancia [2].
El presente trabajo trata de cuantificar los 16 EPAPAH en los productos de pirólisis de purín de cerdo, lo que permitirá evaluar su distribución entre las distintas fases producto: sólido, líquido y gas obtenidos en la pirólisis.

\section{Método experimental}

Los experimentos se realizaron a tres temperaturas finales de pirólisis 625,725 y $825 \mathrm{~K}$, en un reactor de lecho fijo el cual se cargaba con un peso de purín de $300 \mathrm{~g}$. Se hizo uso de una corriente de nitrógeno como gas de arrastre que se introducía a la instalación con un caudal de $250 \mathrm{mLN} / \mathrm{min}$. El reactor se calentaba hasta la temperatura de pirólisis con una rampa de calentamiento de $8^{\circ} \mathrm{C} / \mathrm{min}$, y una vez alcanzada la temperatura, se mantenía constante durante 30 minutos para la realización del experimento.

El líquido obtenido en la pirólisis se retenía en dos condensadores a $0^{\circ} \mathrm{C}$, un precipitador electrostático $\mathrm{y}$ un filtro de lana de vidrio, con el fin de asegurar tener el gas lo más limpio posible antes de su análisis. A continuación, una resina (Resina XAD2) se encargaba de retener los EPA-PAH presentes en el gas. La composición de los gases de salida se determina con un cromatógrafo de gases (Agilent MicroGC $3000^{\mathrm{a}}$ ) que consta de dos columnas independientes, una de tipo plot $\mathrm{U}$ y otra de tipo tamiz molecular, y de dos detectores de conductividad térmica (TCD). Como gases portadores se utilizan helio y argón para cada columna respectivamente. Los gases analizados son $\mathrm{CO}_{2}, \mathrm{CH}_{4}, \mathrm{C}_{2} \mathrm{H}_{6}, \mathrm{C}_{2} \mathrm{H}_{2}, \mathrm{H}_{2} \mathrm{~S}, \mathrm{H}_{2}, \mathrm{~N}_{2}$.

Una vez se finalizados los experimentos se realizaba una extracción Soxhlet, durante 24h, tanto del producto sólido obtenido o char, como de la resina y testigo resultante. Posteriormente las muestras eran concentradas en un rotavapor. Finalmente, para la 
determinación de los PAH's de las muestras se utilizó un cromatógrafo gases-masas (Cromatografo de gases Agilent 6890A acoplado a un detector selectivo de masas Agilent MSD 5975C equipado con una columna capilar Agilent technology DB$17 \mathrm{M}_{\mathrm{S}}(60 \mathrm{~m} \times 0.25 \mathrm{~mm}$ ID x $0.25 \mu \mathrm{m})$ [3].

\section{Resultados}

Las Figuras 1 y 2 muestran los resultados del experimento realizado a $825 \mathrm{~K}$. La Figura 1 muestra la evolución de los gases de salida del reactor. El nitrógeno (gas inerte) disminuye, conforme el experimento avanza, alcanzando un valor mínimo en el minuto 15, mientras que el resto de compuestos aumentan su concentración. Se obtiene una gran cantidad de $\mathrm{CO}_{2}$ e $\mathrm{H}_{2}$, alcanzando ambos un máximo en el minuto 11 y 18 respectivamente, como consecuencia del proceso termoquímico que se está desarrollando.

La Figura 2 representa las distribuciones individuales totales de los 16 EPA-PAH en las muestras obtenidas tras el experimento. Se puede observar que se detectó la presencia de los 16 EPA$\mathrm{PAH}$, obteniéndose principalmente en los productos sólido y líquido de la pirólisis. En el líquido de pirólisis se han encontrado los 16 EPA-PAH, existiendo compuestos tanto ligeros como pesados. En el caso del sólido, quedan retenidos únicamente fenantreno, fluorantreno y pireno, todos ellos de masa intermedia, obteniéndose una concentración elevada de estos tres. En el gas predomina el naftaaleno debido a ser el compuesto más ligero. También existen compuestos más pesados en el gas, aunque la concentración de todos ellos es muy pequeña (aproximadamente 100 veces menor que en líquido y sólido de pirólisis).

\section{Conclusiones}

Los EPA-PAH formados en la pirólisis de purín de cerdo se encuentran presentes en todos los productos, gas, líquido y sólido.

Los EPA-PAH formados se retienen en mayor proporción en el líquido de pirólisis, pudiendo encontrarse en éste los 16 EPA-PAH. En el char únicamente se detectaron fenantreno, fluorantreno y pireno, que están presentes en concentraciones elevadas. El gas de pirólisis se obtiene bastante limpio, con pequeñas concentraciones de EPA$\mathrm{PAH}$, siendo los compuestos ligeros los presentes en mayor concentración.

\section{Agradecimientos}

Los autores agradecen a la Universidad de Zaragoza (UZ) y al Centro Universeitario de la Defensa (CUD) por la financiación recibida (UZCUD2017TEC-01). I. Adánez-Rubio agradece al MINECO y a la Universidad de Zaragoza (UZ) por la beca postdoctoral obtenida (FJCI-2015-23862).

\section{REFERENCIAS}

[1]. H. RICHTER, J.B. HOWARD* . Formation of polycyclic aromatic hydrocarbons and their growth to soot - a review of chemical reaction pathways. Progress in Energy and Combustion Science 26 (2000) 565-608.

[2]. US Environmental Protection Agency EPA. Health assessment document for diesel engine exhaust; 2002. Available on-line: http://cfpub.epa.gov/ncea/cfm/recordisplay.cfm?deid $=29060$

[3]. NAZLY E. SÁNCHEZ, JESÚS SALAFRANCA, ALICIA CALLEJAS, ANGELA MILLERA, RAFAEL BILBAO and MARIA U. ALZUETA. Quantification of polycyclic aromatic hydrocarbons (PAHs) found in gas and particle phases from pyrolytc processes using gas chromatography-mass spectrometry (GC-MS). Fuel. 2013, 107, 246-53.

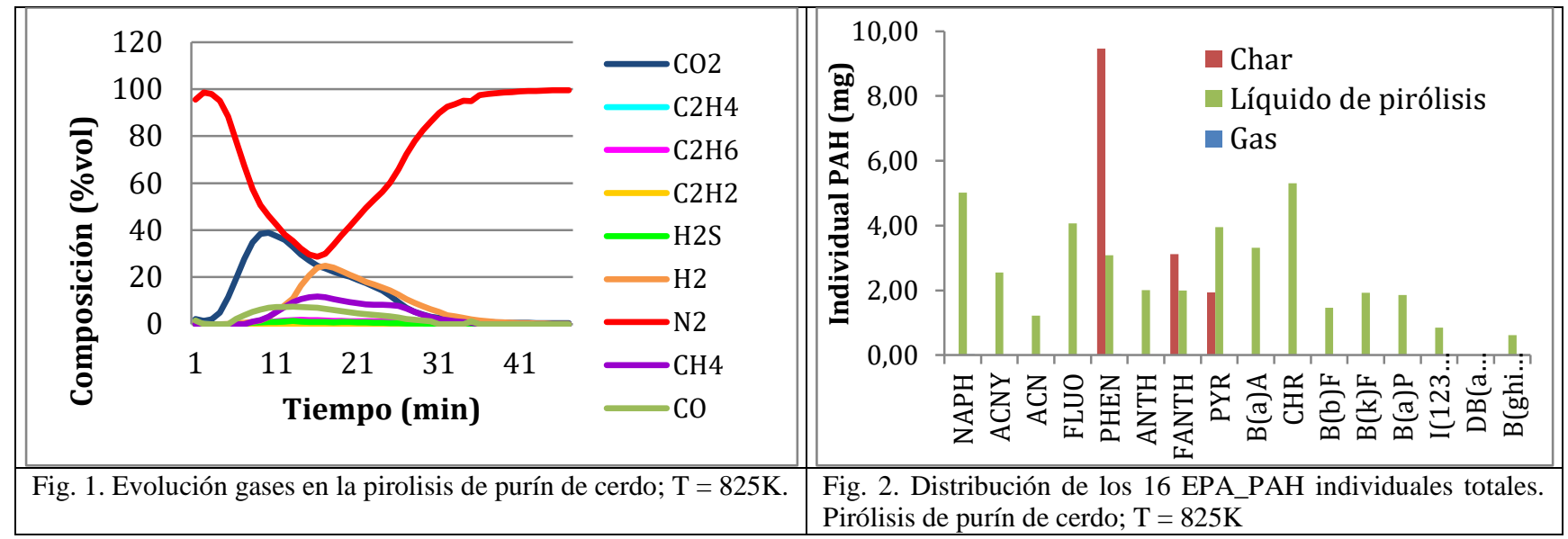

Revista "Jornada de Jóvenes Investigadores del I3A", vol. 6 (Actas de la VII Jornada de Jóvenes Investigadores del I3A - 8 de junio de 2018). ISSN 2341-4790. 